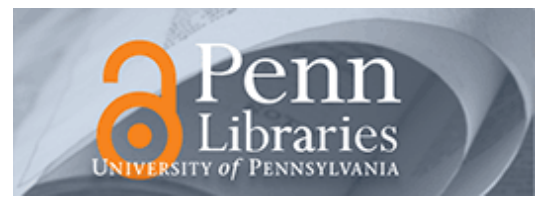

University of Pennsylvania

ScholarlyCommons

Operations, Information and Decisions Papers

Wharton Faculty Research

$2-2004$

\title{
The Copy-Exactly Ramp-Up Strategy: Trading-Off Learning With Process Change
}

Christian Terwiesch

University of Pennsylvania

Yi Xu

Follow this and additional works at: https://repository.upenn.edu/oid_papers

Part of the Mathematics Commons

\section{Recommended Citation}

Terwiesch, C., \& Xu, Y. (2004). The Copy-Exactly Ramp-Up Strategy: Trading-Off Learning With Process Change. IEEE Transactions on Engineering Management, 51 (1), 70-84. http://dx.doi.org/10.1109/

TEM.2003.822465

This paper is posted at ScholarlyCommons. https://repository.upenn.edu/oid_papers/227

For more information, please contact repository@pobox.upenn.edu. 


\title{
The Copy-Exactly Ramp-Up Strategy: Trading-Off Learning With Process Change
}

\begin{abstract}
Production ramp-up is the period of time during which a manufacturing process is scaled up from a small laboratory-like environment to high-volume production. During this scale-up, the firm needs to overcome the numerous discrepancies between how the process is specified to operate as written in the process recipe and how it actually is operated at large volume. The reduction of these discrepancies, a process that we will refer to as learning, will lead to improved production yields and higher output. In addition to its learning effort, however, the firm also attempts to change the process recipe itself, which can be in direct conflict with the learning objective. We formalize this intertemporal tradeoff between learning and process change in the form of a dynamic optimization problem. Our model explains the idea of a "copyexactly" ramp-up, which freezes the process for some time period, i.e., does not allow for any change in the process. Mathematically, this corresponds to a process improvement policy which delays process changes, thereby exhibiting a nonmonotone trajectory, which we show to be optimal if the initial knowledge level is low, the lifecycle short and demand growth is steep, and learning is difficult.
\end{abstract}

\section{Disciplines \\ Mathematics}




\title{
The Copy Exactly Ramp-up Strategy: Trading-off Learning with Process Change
}

\author{
Christian Terwiesch and Yi Xu
}

August 4, 2003

\begin{abstract}
Production ramp-up is the period of time during which a manufacturing process is scaled up from a small laboratory-like environment to high volume production. During this scale-up, the firm needs to overcome the numerous discrepancies between how the process is specified to operate as written in the process recipe and how it actually is operated at large volume. The reduction of these discrepancies, a process that we will refer to as learning, will lead to improved production yields and higher output. In addition to its learning effort, however, the firm also attempts to change the process recipe itself, which can be in direct conflict with the learning objective. We formalize this inter-temporal trade-off between learning and process change in form of a dynamic optimization problem. Our model explains the idea of a "copy-exactly" ramp-up, which freezes the process for some time period, i.e. does not allow for any change in the process. Mathematically, this corresponds to a process improvement policy which delays process changes, thereby exhibiting a non-monotone trajectory, which we show to be optimal if the initial knowledge level is low, the lifecycle short and demand growth is steep, and learning is difficult.
\end{abstract}




\section{Introduction}

Many high-tech industries are struggling in environments of shrinking product lifecycles and increasingly complex production technologies. The window for selling many products has shrunk to less than a year in industries such as semiconductors, disk-drives and telecommunications. These market dynamics pressure production facilities to begin fullscale production at a point when the underlying process technology is still ill understood. As a result, firms suffer from substantial yield losses, which can dramatically affect the economics of the product, production facility, and business.

We define production ramp-up as the period of time following the introduction of a new process into a production facility with the objective to scale up production output from the small batches used in laboratory environments to the large volumes requested by the market. A recent example of the importance of yield losses during ramp-up can be found in AMD's efforts to compete with INTEL in the microprocessor market. AMD had several generations of products that were slow to ramp, leading to limited market acceptance and financial difficulties for AMD. More recently, INTEL experienced problems ramping up the yield of its .18 micron version of the Pentium. Industry observers report that it was this effective ramp-up of AMD's K7 processor which allowed AMD to gain market share in the high end segment of the PC market (Electronic Buyers' News, June 21, 1999).

Given the "premature" transition of the product from the R\&D labs into commercial production, a company finds itself in a difficult situation. On the one hand, it wants to begin accumulating knowledge with the newly introduced process, in an attempt to overcome the numerous discrepancies between how the process should be operated - as outlined in the process recipe (process specifications) - and how the process is actually operated in the production facility. The reduction of these discrepancies, a process that Zangwill and Kantor (1998) refer to as waste reduction or more generally as learning, will 
lead to improvements in yields.

On the other hand, the firm wants to further refine the current process recipe, a phenomena that we will refer to as process change or recipe modification. Such refinements might be based on technologies created outside the firm, leading to potential acquisitions of new production equipment, upgrades of software, and increases in automation, or it might come from internal efforts including research and development and process engineering. Following such a recipe modification, the firm moves to a new performance trajectory, with a more desirable frontier, which we model as lower unit costs. While beneficial in the long-run, a recipe modification during ramp-up provides a disruption in the firm's learning process: routines that were just developed in an attempt to fully implement the old recipe become outdated and new or revised routines are required.

In this article we model the tension between learning and process change, leading to the following novel contributions. First, we extend the literature on normative learning models and apply them to the problem of production ramp-up, which has been identified as an important blank space on the map of product development research (Krishnan and Ulrich 2001). We describe the ramp-up strategy of copy exactly, which is a real-world phenomena of substantial importance, and relate it to models of learning and process change. Second, we explicitly model the tension between process change and learning. Contrary to most of the earlier work (a notable exception is the article by Carillo and Gaimon 2000), we show that it can be optimal to delay process change, even if change would be for free. Third, our paper differs from Carillo and Gaimon's work as we explicitly capture the details of how change leads to disruption. In the Carillo and Gaimon work, change causes a short term capacity reduction, but does not impact knowledge itself. Our formulation, in contrast, takes a more detailed and micro-level perspective as we model the effect of the control variable 'process change' on the state variable 'knowledge' directly in the optimization problem. 
Our theoretical contributions have direct managerial implications. We explain the concept of a "copy-exactly" ramp-up. This ramp-up strategy, which is extremely conservative with respect to process change, was introduced by INTEL for their ramp of new fabs around 1991, and has since then been augmented to the company's fundamental rampstrategy. Remarkably, while almost every member of the semiconductor industry is aware of copy-exactly, most competitors do not "buy into it", and favor a much more aggressive process change during the ramp. Moreover, several suppliers and industry observers argue that the recent changes in the semiconductor landscape - including 300mm wafers, shorter product lifecycles and further shrinking line-widths - make copy-exactly an outdated rampstrategy. But what is the influence of these variables on the choice of copy-exactly, or a process change policy in general? How does the complexity of the process or the length of the product lifecycle influence these decisions? Our analysis reveals several structural results providing answers to these questions, including that copy-exactly is beneficial if the initial understanding of the process is low, the lifecycle is short and grows quickly, the process is difficult to improve and sensitive to even small modifications.

\section{Theoretical Framework and Industrial Examples}

We focus on production ramp-ups of high-tech products, such as semiconductors, disk drives, and complex pharmaceutical compounds. Given the short lifecycles and rapid price erosion in these industries, management is often forced to bring products to market before the manufacturing process is fully understood. Given the joint pressure from both, market and technology, and the resulting rate of change, management cannot afford to optimize and fine-tune all product and process related decisions prior to launch. Instead, it has been reported that in such "high velocity" environments firms have to rely on an experiential problem-solving strategy (Eisenhardt and Tabrizi 1995). From an operations 
management perspective, such an approach leads to low stages of knowledge at the outset of production, which can cause substantial yield losses (Terwiesch and Bohn 2001).

Yields are an important performance measure during production ramp-up because they have a major effect on process economics (McIvor et al. 1997). Low yields reflect gaps between how the process should be operated, as specified in the process recipe, and how the process is actually operated in the production facility. Pisano (1995) reports how pharmaceutical companies experience yield losses when they transfer a complex product from an R\&D lab to commercial production. The process recipe is created in an environment of highly trained workers, small production batches, and extremely sophisticated production equipment. In contrast, facilities used for commercial production are operated by regular work-force, they produce large quantities leading to so called scale-up problems ${ }^{1}$, and use equipment frequently incapable of achieving the tight tolerances defined by the process recipe. Thus, the phase of production ramp-up is primarily one of yield improvement during which the firm attempts to accurately implement the current process recipe at a large scale. We refer to these efforts as learning or waste reduction and discuss them in the following subsection.

\section{Implementing the Current Process Recipe: Learning and Waste Reduction}

Learning describes a firm's accumulation of knowledge and its movement along a certain trajectory, called the learning curve. Learning curves have received a great deal of attention in the manufacturing and - more recently - organizational literature (Argote and Epple 1990). The traditional learning curve model postulates that variable production costs fall as the logarithm of cumulative production (Wright 1936, Levy 1965). More recently, the same argument has been applied to other performance measures, including production

\footnotetext{
${ }^{1}$ Scale-up problems in chemical reactions can be extremely complex and non-linear. For example, when producing $10 \mathrm{~kg}$ of a substance opposed to $10 \mathrm{~g}$, it is not sufficient to multiply all specificaions with a factor 1000.
} 
yields (Hatch and Mowery 1998).

In addition to the traditional argument that learning is driven by cumulative output, numerous factors that can explain why and how fast a firm moves down a learning curve have been identified. For example, Lapré et al. (2001) relate learning to the cumulative number of quality improvement projects under taken at a certain point in time. A similar position has been taken by Hatch (2002), who relates human resource practices in semiconductor manufacturing to performance improvement. The importance of training in learning curves has been discussed by Anderson (2001) and modeled formally by Carrillo and Gaimon (2000).

Others have argued that learning is primarily driven by the proportion of production capacity that can be managed towards generating knowledge, as opposed to output. This follows the notion of a learning laboratory (Bohn 1987) and has been observed empirically by Pisano (1995) during the ramp-up of biotechnology processes. Terwiesch and Bohn (2001) as well as Chand et al. (1996) analyze the resulting trade-off between exploiting an organizations current capacity towards generating revenues, versus using capacity to explore opportunities for process improvement ${ }^{2}$.

Despite these differences in perspective of what actually drives learning at the microlevel, three elements are common across the literature. First, learning is a process of accumulation, specifically one of building an increasing base of knowledge and organizational routines. This view underlies the econometric estimation of learning rates, which

\footnotetext{
${ }^{2}$ Many others have looked at learning and thereby the rate of performance improvement at a more aggregate level. For example, Mody (1989) provides a model, which explicitly examines engineering effort as a driver of learning. Dorroh et al. (1994) have a related model of make to order production, with a production function that takes knowledge and other resources as inputs. Knowledge is produced by an explicit investment in learning, independent of the ongoing production. This approach has also been taken in the operations management literature, where models of process improvement have been presented by Fine (1986) and Fine and Porteus (1989).
} 
use cumulative output, cumulative investment, or cumulative learning activity, as an independent variable as well as the existing mathematical models. Second, while a firm would always prefer a large knowledge base over a small knowledge base, learning does not come for free. Costs of learning obviously depend on the sources of learning, and can include short-term disruptions through training (Carrillo and Gaimon 2001), capacity investments (Chand et al. 1996), or mere financial investments (Fine and Porteus 1986). Third, there is an overall agreement that the returns to investment in learning are diminishing. This is consistent with Zangwill and Kantor's model of waste reduction, which assumes that each halving of waste requires a roughly constant amount of effort (Zangwill and Kantor 1998). In our models, zero waste equates to $100 \%$ yield. Figure 1 summarizes these three common points.

\section{Recipe Modifications: Improvement Potential and Disruption of Learning}

Achieving a perfect implementation of the process recipe, which basically corresponds to eliminating all waste from the process, is not the only source of process improvement. After the process recipe has been defined and transferred into commercial production, new improvement opportunities typically arise. This is especially likely if the product has to be rushed to market, thereby requiring process engineering to "cut some corners" in the specification of the process recipe ${ }^{3}$.

Although the firm might benefit from modifications in the process recipe after product transfer in the long-run, process change can be the source of serious short-term disruptions (Carrillo and Gaimon 2000). In complex production processes, even slight recipe modifications can make millions of dollars in waste reduction effort obsolete and require the development of new knowledge and routines. New technologies also directly affect the

\footnotetext{
${ }^{3}$ Our focus is purely on changes in the process recipe opposed to changes in the actual product. See Bhattacharya et al. (1998) for a model describing the situations under which it is optimal to freeze the product specifications early vs. late.
} 
productivity of the firm's workforce, as argued by Gaimon (1997). In that sense, a modification of the current process recipe actually reduces (relevant) knowledge. This does not mean that the organization actually forgets what it has learned before, but rather that what it has learned in the past has partially lost its relevance and that there are additional things to be learned ${ }^{4}$.

Many models of learning have ignored the difference between learning and process change. The amount of accumulated knowledge by itself is insufficient to accurately describe the production process during ramp-up, as it only captures how closely the firm is able to execute the current process recipe, as opposed to including information about the quality of the process recipe itself. To capture this additional dimension, a second state variable is needed.

To help illustrate the need for this distinction, consider a person who wants to master the art of playing the piano. In this case, learning corresponds to the person practicing. Practicing is typically based on a specific piece of music the person plays. Initially, this would be a very simple piece. While practice might make the person perfect in playing this specific piece, the first time the person switches to a more advanced piece would not sound all too attractive. Following the switch to the more advanced piece, the person again improves based on practice. Overall, the process of mastering the piano is a subtle interplay of practicing a piece versus advancing to more sophisticated levels. To capture this interplay, we need to keep track of what piece the person is currently playing (Bach vs. children's music) and how much the person practiced this piece and other pieces before.

The need for a second state variable also becomes apparent when looking at empirical studies who have reported cases where an organization invests in learning, but does not

\footnotetext{
${ }^{4}$ This is consistent with Lapré et al. (2000), who show in a steel chord manufacturing plant that certain projects, namely those that were started out of the corporate $R \& D$, actually decreased process performance and caused significant disruption in the production process.
} 
change its process recipe. This situation is prevalent during the ramp-up of a new production process (McDonald 1999, Hatch and Mowery 1996). Based on this argument, we extend Figure 1 by introducing a second state variable (Figure 2). The firm, thus, faces a trade-off in deciding about its investment in learning and about the amount of process change.

\section{Industrial Examples}

Chris McDonald - director of the Advanced Technology Development Facility at INTEL - describes the company's approach to production ramp-up in the Intel Technology Journal (Q4 1998). He observes that engineers have a desire for modifying the process recipe, especially during production ramp-up. The emphasis under the copy-exactly approach, in contrast, is to first focus on the implementation of the existing process recipe. Once the new process achieves high yields in high volume, production line engineers can take over and conduct modifications. This is an example where - during the ramp-up - a firm invests in learning (yield improvement) but explicitly avoids modifications. Modifications are risky, as they "make the fab behave differently, with different sensitivities, process differences and interactions pertaining specifically to wafer fabrication equipment, which may lead to a detrimental impact on yield (INTEL document)".

In addition to recipe modifications in the strict sense (e.g. a change in temperature at a diffusion furnace), process change can also relate to equipment acquisition. INTEL insists on having the exact same equipment for the high volume production as was used in the process development. While this reduces the company's flexibility in benefiting

from recent supplier developments, it does facilitate the transfer from the development fab to other production facilities. This is illustrated in Figure 3: in the left part of the Figure, the firm acquires the latest equipment for the beginning of production in Factory 1. However, as this provides a substantial process change, yields drop following the transfer to 
volume production, leading to direct economic losses and the need for additional learning investments.

Under copy-exactly (right part of the Figure), INTEL uses the same equipment in volume production as it did in the development fab (i.e. no process change), which allows the firm to continue along the yield curve of the development fab. However, with copy-exactly, INTEL is forced to acquire "old technology" for its volume production facilities. Bernie Wood, director of marketing at an equipment vendor, remarks: "They [INTEL] use some of the oldest equipment in the business, some of the most outdated equipment, because of their copy-exactly requirements (Electronic Business Asia, September 2000)". The "idealized" time series in the upper part of Figure 3 are strongly supported by empirical data. The lower part of Figure 3 contrasts the ramp of the 1.0-micron technology, the last ramp at INTEL not following copy-exactly, with the one of the 0.5-micron technology using the copy-exactly approach and reveals the same pattern as in the upper part. A similar observation is reported by Thomke (1999).

\section{Model Formulation}

Based on the theoretical framework presented in Figure 2, we now present a profit maximization model that captures the interplay between recipe modifications (process change) and learning during production ramp-up. Following the earlier research by Spence (1981), Fine (1986), Chand et al. (1996), Li and Rajagopalan (1998), and Carrillo and Gaimon (2000), we use a deterministic model with continuous state and decision variables. This approach provides an aggregate view of the detailed dynamics of learning and does not consider the micro-level uncertainties engineers encounter in their daily work ${ }^{5}$. A summary of the model notation and the underlying assumptions are provided by Tables 1 and

\footnotetext{
${ }^{5}$ See Fine and Porteus (1989) for a stochastic model of the dynamics of process improvement.
} 
2 respectively.

Let $0 \leq q(s) \leq \bar{q}$ be the process change rate at time $s \in[0, t]$, where $\bar{q}$ is the maximum amount of process change at any time. Then, for any given time $t$, the cumulative amount of process change, $Q(t)$, can be expressed as

$$
Q(t)=Q(0)+\int_{0}^{t} q(s) d s
$$

The cumulative change in process recipe, $Q(t)$, can be viewed as a state variable that represents the production facility's performance potential with a given process recipe. Differentiating both sides of (1) with respect to $t$, we obtain the following differential equation that governs the motion of state variable, $Q(t)$ :

$$
\dot{Q}(t)=q(t)
$$

Let $x(t)$ denote the knowledge level of the process at time $t$ for the process recipe defined by $Q(t)$. Following Figure 2, knowledge is increasing in the cumulative investment the firm made in learning and decreasing with every modification in the process recipe. Let $0 \leq v(s) \leq \bar{v}$ be the learning effort the firm invests at time $s \in[0, t]$, where $\bar{v}$ is the maximum amount of effort that can be invested in learning at a point in time. Thus, the knowledge level at time $t, x(t)$, can be expressed as:

$$
x(t)=x(0)+\alpha \int_{0}^{t} v(s) d s-\beta \int_{0}^{t} q(s) d s
$$

where $\alpha \geq 0$ and $\beta \geq 0$ are the relative weights on the impact of learning and process change respectively. We can think of $\alpha$ being a measure of the speed of learning, be it in the form of the engineering talent, the quality of measurement equipment guiding the learning, or simply the "learnability" of the process itself. $\beta$ can be labelled as the disruption parameter, as it indicates to what extend the firm suffers a loss in knowledge for a unit of process change. It thereby reflects the complexity of the production process and the degree of interdependence between process steps. 
Differentiating both sides of (3) with respect to $t$, we obtain the following differential equation governing the motion of the state variable, $x(t)$ :

$$
\dot{x}(t)=\alpha v(t)-\beta q(t)
$$

Following Figure 2, our model is built around two state variables, the performance potential of the process recipe $Q(t)$ and knowledge level $x(t)$. Their motion is governed by the differential equations (2) and (4), respectively.

At time 0 , the firm starts to ramp-up the new process to produce a new product with a lifecycle duration of $T$. In presence of an imperfect implementation of the process recipe, the firm will not be able to fully benefit from its nominal capacity level. Instead, it will suffer from yield losses, meaning that some of the obtained output will not be of sufficient quality to be sold to the market ${ }^{6}$. We assume process yield at time $t, y(x(t))$, to be an increasing and concave function of the knowledge level $x(t)$. That implies $y^{\prime}(x(t)) \geq 0$ and $y^{\prime \prime}(x(t)) \leq 0$. At the abstract level, yield losses can be interpreted as waste. Consequently, assumption A1 is consistent with the analytical work by Zangwill and Kantor (1998) and the empirical work by Lapre et al. (2001). Note that $0 \leq y(t) \leq 1$. Furthermore, we assume that $y(0)=0$ and $y(\infty)=1$, i.e. yields approach $100 \%$ with knowledge reaching perfection.

There are three kinds of costs in the system, unit manufacturing cost, cost of learning, and cost related to recipe modifications. The unit manufacturing cost at time $t, c(Q(t))$, is assumed to be a decreasing and convex function of the process recipe at time $t, Q(t)$, which is defined in equation (1). That implies $c^{\prime}(Q(t)) \leq 0$ and $c^{\prime \prime}(Q(t)) \leq 0$. We focus on modifications related to the process; a similar analysis holds for modifications related to

\footnotetext{
${ }^{6}$ As we do not explicitly consider rework in our model, the reader should think of $y(t)$ being the composite yield, which includes the units passing inspection in their first attempt and units passing inspection after rework (see e.g. Bohn and Terwiesch 1999 for various definitions of production yields).
} 
the product, which are associated with higher prices (Roth et al. 1994). At first sight, there seems to be an inconsistency between our model formulation and the traditional learning curve literature, which has postulated that the cost of production decreases with learning. To clarify what seems like an inconsistency, the following observations are helpful. The cost per good unit can be computed as $c(Q(t)) / y(x(t))$. From this expression, we can observe that the cost per good unit is decreasing with learning. At the abstract level, one can think of the cost of a good unit as being broken up into a knowledge depending part (which we call yield) and a process change dependent part ${ }^{7}$. Thus, parts of the cost are impacted by process change and other parts are impacted by learning. Both, change and learning, decrease the costs of a good unit, thus A1 and A2 are in line with existing research.

Let $k(t)$ be the firm's production rate at time $t$. Thus, $y(x(t)) \times k(t)$ units of good products will be sold to the market. The firm faces a downward sloping demand curve represented by $p(y(x(t)) k(t))$. Hence, the realized final revenue at time $t$ will be equal to $y(x(t)) k(t) \times p(y(x(t)) k(t))$. For notational convenience, we denote the revenue as an increasing function of $y(x(t))$ and $k(t)$ that is defined as $R(y(x(t)), k(t))=y(x(t)) k(t) \times$ $p(y(x(t)) k(t))$. Furthermore, we assume the revenue function to be concave and supermodular in $y(x(t))$ and $k(t)$. That implies $R_{y}=\frac{\partial R(y(x(t)), k(t))}{\partial y(x(t))} \geq 0, R_{k} \geq 0, R_{y y} \leq 0, R_{k k} \leq 0$, $R_{y k} \geq 0$ and $R_{k y} \geq 0$. The concavity and the supermodular assumption (assumption A3) has been made by prior research, including Spence (1981), Fine (1986), Chand et al. (1996), Li and Rajagopalan (1998), and Carrillo and Gaimon (2000). The computational Assumptions A4-A6 are also consistent with these earlier studies.

Also, we define $c(0)=\bar{c}>0$ and $c(\infty)=\underline{c}>0$, where $\bar{c}>\underline{c}$. The gap between $\bar{c}$ and $\underline{c}$ can be viewed as the process improvement potential resulting from process change. The

\footnotetext{
${ }^{7}$ This view is consistent with the economist's idea of a Cobb-Douglas production function, which also breaks up the cost of production into multiplicative elements.
} 
learning cost $c_{2}(v(t))$ is assumed to be an increasing and convex function of the units of learning efforts. Therefore, $c_{2}^{\prime}(v(t)) \geq 0$ and $c_{2}^{\prime \prime}(v(t)) \geq 0$. Learning cost reflect the investment associated with the reduction of waste, i.e. efforts that focus on perfecting the current process recipe. Finally, the costs of process change are also assumed to be an increasing and convex function, i.e. $c_{1}(q(t))$, where $c_{1}^{\prime}(q(t)) \geq 0$ and $c_{1}^{\prime \prime}(q(t)) \geq 0$, including expenditures related to equipment upgrades or engineering hours for process development.

The firm's decisions are the production rate $k(t)$, the amount of process change $q(t)$, and learning effort $v(t)$ that are chosen over time to maximize the total profit on the planning horizon $[0, T]$. The firm's ramp-up problem can be formulated as the following continuous time optimal control model ${ }^{8}$.

$$
\begin{gathered}
\max _{\substack{0 \leq k(t) \leq \infty \\
0 \leq v(t) \leq \bar{v} \\
0 \leq q(t) \leq \bar{q}}} J=\int_{0}^{T}\left\{R(y(x(t)), k(t))-c(Q(t)) k(t)-c_{1}(q(t))-c_{2}(v(t))\right\} d t \\
\text { s.t. } \quad \dot{x}(t)=\alpha v(t)-\beta q(t) \\
\dot{Q}(t)=q(t) \\
x(t) \geq 0 \\
Q(t) \geq 0 \\
x(0)=x_{0} \geq 0 \text { and } Q(0)=0
\end{gathered}
$$

Our analysis does not include a discount factor, as most of the analysis becomes analytically extremely complex, without leading to any additional insights (see Carillo and Gaimon 2000 for a similar observation).We found numerically that all our results continue to hold if a discount factor is introduced (See section 6).

\footnotetext{
${ }^{8}$ In this model, we do not consider any salvage values of the knowledge or process capability occurring at the end of the planning horizon. The impact of salvage value is well understood and has been studied by e.g. Dorroh et al. (1994) and Carillo and Gaimon (2000).
} 
Throughout the article, we use dots to denote derivatives with respect to time and primes to denote derivatives with respect to decision variables. Constraints (6) and (7) are the state motion governing equations for these two state variables, respectively. Constraints (8) and (9) ensure the non-negativity of the state variables. Constraint (10) specifies the starting points of the state variables.

Our model is closely related to the work by Carrillo and Gaimon (2000), who also discuss the disruptive effect of process change. In their model, the disruptive effect of process change takes the form of a short term capacity loss (similar to Terwiesch and Bohn 2001) but is assumed to increase knowledge. In contrast, in our model, process change does not generate knowledge automatically (e.g., upgrading to a faster diffusion furnace does not create direct knowledge about its appropriate usage), but merely shifts the frontier of the underlying technology. In this setting, the disruptive effect of process change is one of lower process yields (e.g. the upgraded diffusion furnace requires different temperature settings) and additional learning effort is required to restore yields to the previous level.

\section{Analytical Results}

Our first result, Theorem 1, characterizes the optimal learning policy and the optimal process change policy. Both policies are so-called threshold policies, which are common in models of dynamic control.

Theorem 1 (Optimal Learning and Process Change Policies): The optimal learning and process change policies can be characterized as the following,

(1) For any given time $t$, there exist $x_{1}(t) \leq x_{2}(t)$ such that $v^{*}(t)=\bar{v}$ if $x(t) \leq$ $x_{1}(t), v^{*}(t) \in(0, \bar{v})$ if $x_{1}(t) \leq x(t) \leq x_{2}(t)$, and $v^{*}(t)=0$ if $x(t) \geq x_{2}(t)$. 
(2) At any time $t$, for any given $Q(t)$, there exist $x_{a}(Q(t), t) \leq x_{b}(Q(t), t)$ such that $q^{*}(t)=0$ if $x(t) \leq x_{a}(Q(t), t), q^{*}(t) \in(0, \bar{q})$ if $x_{a}(Q(t), t) \leq x(t) \leq x_{b}(Q(t), t)$, and $q^{*}(t)=\bar{q}$ if $x(t) \geq x_{b}(Q(t), t)$

The optimal learning policy only depends on one state variable, the knowledge level $x(t)$. This is because the learning effort only affects the knowledge level of the process (see equation (6)). In contrast to the optimal learning policy, the optimal process change policy depends on both state variables, the knowledge level and the cumulative process change. This difference results from the fact that the process change rate affects both state variables in the system (see equations (6) and (7)). Therefore, when making decisions related to process change, one needs to consider the current knowledge level (How well is the process understood?) as well as the cumulative amount of process change (How advanced is the current process?). The optimal amount of process change balances the revenue and cost effects caused by the state variables.

According to the optimal process change policy, more aggressive process change is more desirable when the knowledge level of the current process is above a certain level. A higher knowledge level builds a good foundation for more aggressive process change in the future, because the negative effect of decreasing knowledge caused by process change at a higher knowledge position is less than the one at lower knowledge position. This is summarized by Corollary 1.

Corollary 1 (Impact of initial knowledge level, $x(0)$ ): (a) A firm with lower initial knowledge level will invest more in learning and will pursue less process change.

(b) A firm with lower initial knowledge level will start process change later.

The policy outlined in Corollary 1 relates to Jaikumar and Bohn's framework of the "stages of knowledge": the firm is only allowed to modify the current process recipe if it is able to fully implement the current one. It is required "to be able to walk before 
attempting to run".

Our second Corollary postulates that the knowledge threshold increases with cumulative process change. That simply implies that the more advanced the process, the less change should be done to it, which is a natural consequence of the convexity assumption of the unit manufacturing cost function $c(Q(t))$.

Corollary 2 (Monotonicity of the optimal process change policy):

$$
\text { If } Q_{1}(t) \geq Q_{2}(t) \text {, then } x_{a}\left(Q_{1}(t), t\right) \geq x_{a}\left(Q_{2}(t), t\right) \text { and } x_{b}\left(Q_{1}(t), t\right) \geq x_{b}\left(Q_{2}(t), t\right) \text {. }
$$

The result of Corollary 2 differs from the results outlined in the existing literature. In most of the prior work, the optimal policies assume that the control variables (learning) have a positive effect on the one state variable (Figure 1 vs. Figure 2). Thus, the only reason for not having an infinite rate of knowledge accumulation lies in the costs associated with the control variables. Our model introduces a second trade-off in addition to the one related to cost. Even if changes in the process recipe were free, they might not be desirable as they reduce knowledge in the short term.

Theorem 2 (Optimal Strategies): (1) The optimal learning investment, $v^{*}(t)$, decreases over time.

(2) If $\beta\left[R_{y} y^{\prime}(x(t))-\dot{\eta}(t)\right]+c^{\prime}(Q(t)) k(t)>(<) 0$, the optimal process change rate, $q^{*}(t)$, increases (decreases) over time.

(3) When $\dot{x}(t) \geq 0$, the optimal production rate, $k^{*}(t)$, increases over time; When $\dot{x}(t)<$ 0 , the optimal production rate, $k^{*}(t)$, increases (decreases) over time, if $R_{k y} y^{\prime}(x(t)) \dot{x}(t)-$ $c^{\prime}(Q(t)) q(t) \geq(\leq) 0$.

The decreasing amount of investment described in Theorem 2.1 is consistent with results from existing models. Theorem 2.2 describes the specific pattern of the optimal process change rate will depend on the relative positions of the state variables, $x(t)$ and $Q(t)$. This structural result is consistent with the findings of Carillo and Gaimon (2000). Especially 
if the underlying production process is extremely complex, it is likely that the initial value of knowledge $x(0)$ is below the corresponding threshold value. In such a case, no process change is allowed and all effort is directed towards mastering the current recipe (i.e. increasing $x(t)$ and thereby production yields). INTEL's copy-exactly philosophy is one illustrative example of this. After the current process recipe is implemented with adequate levels of yields, process change can become beneficial.

The optimal rate of production postulated by Theorem 2.3 can be increasing or decreasing over time. The output rate increases monotonically only in the special case when no process change is introduced (and correspondingly the knowledge level is strictly increasing, $\dot{x}(t) \geq 0$ ). If we consider the disruptive effect of process change leading to phases during the production ramp-up when the knowledge level is decreasing $(\dot{x}(t)<0)$, the optimal production path can be either increasing or decreasing. We see that small reductions in knowledge can still increase the production volume, because the revenue generated by increased production is still greater than the loss associated with lower yield (the right-hand side of the equation can still be positive). However, for larger reductions in knowledge, production volume is scaled down as a large production volume would amplify the yield losses. Only after yields have been increased, is it economical to also increase production rates.

Theorem 3 (Sensitivity Analysis): (1) The higher the learning parameter $\alpha$, the higher is the optimal learning investment rate $v^{*}(t)$. However, the optimal process change rate $q^{*}(t)$ is not monotone in $\alpha$.

(2) The higher the disruption parameter $\beta$, the higher is the optimal learning investment rate $v^{*}(t)$ and the lower is the optimal process change rate $q^{*}(t)$.

As expected, if the costs of learning are low ( $\alpha$ is small), the firm should invest relatively more resources into learning. The relationship between the learning parameter $\alpha$ and the 
optimal process change rate is more complicated though. There are two effects between $\alpha$ and $q^{*}(t)$. On the one hand, a higher $\alpha$, i.e., a higher return from a unit of learning effort, enables the firm to pursue a more aggressive change strategy, as the disruption caused by process change can be more easily recovered through future learning investments. On the other hand, process change by itself can be costly, and might not be recovered over the finite planning horizon.

An increase in the disruption parameter $\beta$ increases the short term knowledge loss associated with process change. Even a small amount of process change might be the source of a major reduction in production yields. Thus, it is optimal to implement process change more conservatively. At the same time, the firm should increase its investment in learning to compensate for the knowledge losses caused by future process change.

\section{$5 \quad$ Learning before Doing}

After analyzing the general model, we now turn to a slight variation of our ramp-up problem that we label learning before doing. The case of learning before doing occurs if the learning process does not primarily occur during commercial production, but prior to it, for example in a dedicated pilot plant. Instead of producing salable output, the major focus of such a pilot production facility is to develop a process capability that can then be replicated in the commercial facilities at launch time (Pisano 1995).

We define process capability as the linearly weighted combination of yield and unit manufacturing cost, where the weights are $\theta$ and $\xi$ respectively. Thus, the capability at time $s$ equals $\theta y(x(s))-\xi c(Q(s))$. Basically, the process capability is a measure of process profitability at launch time, since $\theta y(x(s))$ measures the firm's capability of implementing the current process recipe and $\xi c(Q(s))$ measures the quality of the recipe itself.

The costs for learning investment and process change and the dynamics between the 
state variables and the control variables are as defined in the previous section. The only difference lies in the objective function, which is concerned only with the process capability at time $T$, as opposed to its detailed trajectory.

In the example of a person learning to play the piano discussed previously, this corresponds to the case where the person is only concerned about her skills at one event, e.g. one important concert, as opposed to a good performance at every practice session. The learning before doing problem can be defined as follows:

$$
\max _{\substack{0 \leq v(t) \leq \bar{v} \\ 0 \leq q(t) \leq \bar{q}}} J=\theta y(x(T))-\xi c(Q(T))-\int_{0}^{T}\left[c_{1}(q(t))+c_{2}(v(t))\right] d t
$$

s.t. (6), (7), (8), (9), and (10).

We can characterize the optimal learning and process change policies in the following theorem.

Theorem 4 (Optimal R\&D learning and process change policies):

For a R\&D process defined above, suppose $x(t) \geq 0$ for all $t \in[0, T]$, both the optimal learning rate and process change rate remain constant over the whole time horizon, i.e.,

$$
\begin{aligned}
& v(t)=v^{*} \text { for all } t \in[0, T] \\
& q(t)=q^{*} \text { for all } t \in[0, T]
\end{aligned}
$$

where $v^{*}$ and $q^{*}$ solve the equations (35) and (36), respectively.

Theorem 4 suggests a fundamentally different policy for managing the pilot process as opposed to the commercial process (see Theorem 1). During commercial production, management needs to permanently "have an eye on" profits. In a pilot plant however, it can focus purely on knowledge creation. What is the most economical way of increasing performance? Because of the convexity of the cost functions, knowledge is best created by "walking a steady pace". The decisions related to learning and change collapse to a static 
marginal analysis on the trade-off between gains and costs, uncorrupted by commercial pressures during the learning process.

During an R\&D process, managers often face trade-off between process maturity and time-to-market. Reflecting this consideration in our model, we not only need to determine the optimal learning and process change rates, but also need to choose the optimal R\&D length $T$. Assume the penalty cost of delayed market entry is $\Gamma(T)$, which is an increasing and convex function of $T$. We can now extend the objective function (11) to capture the trade-off between rushing the product to market (low $T$, leads to high costs per good unit) and delaying the launch (high $T$, leads to high opportunity cost).

From Theorem 4, we know that for any given $T$, the optimal learning and process change rates stay constant $\left(v^{*}\right.$ and $\left.q^{*}\right)$ over the time. Thus, at the end of the R\&D process, we have $x(T)=\left(\alpha v^{*}-\beta q^{*}\right) T$ and $Q(T)=q^{*} T$. Therefore, for any given $T$, the corresponding objective function can be written as follows,

$$
J(T)=\theta y\left(\left(\alpha v^{*}-\beta q^{*}\right) T\right)-\xi c\left(q^{*} T\right)-\Gamma(T)-\left[c_{1}\left(q^{*}\right)+c_{2}\left(v^{*}\right)\right] T .
$$

Since $y($.$) is concave and c($.$) and \Gamma($.$) are convex, the objective function J(T)$ is concave in $T$. Therefore, the optimal R\&D time duration $T$ is uniquely determined by the first order condition to (12). The first order condition balances the marginal benefit of spending one more unit of time on R\&D and the marginal loss of delaying one more unit time of product launching. Extending this basic model of process maturity, future research is needed to characterize the detailed shape of the opportunity cost $\Gamma(T)$ and how it depends on the competitive market environment. 


\section{Numerical Illustration}

In this section we illustrate our results with a sequence of numerical examples. Unless stated explicitly otherwise, all examples are based on the following functional forms: $y(x(t))=1-e^{\mu x(t)} ; c(Q(t))=\phi+\varepsilon e^{\tau Q(t)} ; c_{1}(q(t))=\gamma q(t)^{2} ;$ and $c_{2}(v(t))=\varpi v(t)^{2}$, where $p(t)$ is the price at time $t$. Therefore, the revenue function can be expressed as $R\left(y(x(t), k(t))=10 \times 5 \times\left(1-e^{\mu x(t)}\right)\right.$. We consider the static price and production rate for our base case. The parameters for our base case are defined as follows: $p(t)=10$; $k(t)=5 ; T=10 ; \alpha=1 ; \beta=1.5 ; \mu=0.05 ; \phi=1 ; \varepsilon=8 ; \tau=0.05 ; \gamma=2 ; \varpi=2 ;$ $Q(0)=0 ;$ and $x(0)=8$.

For these parameter values, our model prescribes a change and learning trajectory as outlined in Figures $4 \mathrm{a}$ and $4 \mathrm{~b}$ respectively. We observe that while the amount of learning investment is a decreasing function over time, this is not true for the optimal change rate. The change rate is increasing for the first three units of time before it peaks and then decreases to 0 at the end of the planning horizon.

Next, we reduce the starting level of knowledge to $x(0)=2$. The resulting trajectories for process change and learning are summarized in Figures 5a and 5b. Figure 5a directly relates to the copy-exactly idea: for the first two units of time, the process is not changed at all, while at the same time, the firm invests heavily into knowledge accumulation. In presence of imperfect knowledge about the production process, the firm shies away from any modification in recipe. Once yields have reached a satisfactory level, modifications are allowed to reduce cost. At the end of the lifecycle an end-of-horizon effect takes over, and the firm myopically harvests its current capability as opposed to building new one.

To better understand this effect, we gradually decrease the starting knowledge from $x(0)=14$ to $x(0)=8,4$, and 2 . The resulting trajectories are depicted in Figures $5 \mathrm{c}$ and 5d. We observe that for high levels of initial knowledge, the change rate indeed resembles 
the learning investment, consistent with the existing literature. However, the lower the initial knowledge, the higher the need for an extended "process freeze". We thus can state the following observation related to copy-exactly (CE) ramp-strategy:

CE-observation 1 (starting knowledge): Production ramp-ups characterized by a low level of starting knowledge will benefit from a CE-ramp.

We now turn to the impact of the learning parameter $\alpha$ and the disruption parameter $\beta$. The corresponding graphs are depicted in Figures $6 \mathrm{a}$ and $6 \mathrm{~b}$ respectively. We observe that greater learnability makes investments in learning more attractive, thereby shifting the optimal learning trajectory upwards. It also allows the firm to absorb / prepare for future process change at lower cost, which in turn increases the rate of process change. Thus, especially for integrated and complex processes, which are typically environments where learning is extremely costly, will a CE-ramp-up be beneficial.

CE-observation 2 (learnability): Production ramp-ups of processes that are very difficult to improve will benefit from a CE-ramp.

A similar observation can be made with respect to the disruption parameter $\beta$, which measures the impact of a change in recipe on production yields. If the process is very sensitive to change and even minor modifications can lead to substantial reduction in yields, the change rate should be slowed down or even stopped completely until a sufficient knowledge base has been accumulated.

CE-observation 3 (disruptions): Production ramp-ups of processes that are extremely sensitive to even small disruptions will benefit from a CE-ramp.

Next, consider how the optimal policies change with the duration of the product lifecycle T. As we discussed above, shorter lifecycles favor myopic action, and lead to a reduction in learning investment. This is illustrated by Figures 7a and 7b.

Shorter lifecycles lead to less process change. This seems obvious concerning the end 
of the lifecycle: the sooner sales will stop, the earlier one should switch to a fully myopic policy. Interestingly though, Figure 7a also depicts a similar behavior at the beginning of the lifecycle. If the lifecycle becomes shorter, early sales are becoming relatively more important, and thus even short periods of low yields can have a substantial impact on the bottom line. As expected, the amount of learning investment is reduced with a shorter lifecycle (see Figure 7b). Taken together, these two effects lead to an early process freeze for extremely short lifecycles, which prompts our fourth CE-observation.

CE-observation 4 (length of lifecycle): Production ramp-ups of processes with shorter product lifecycle will benefit from a CE-ramp.

In many situations, management of a fab or a factory is confronted with an exogenous demand growth trajectory (e.g. driven by a new product diffusion process) that needs to be supported with volume. Consider the Bass diffusion model with the three standard parameters: $m$, the number of potential buyers of the new product, $p$, the coefficient of innovation, and $q$, coefficient of imitation. For a given potential market size $m$, as shown in Figure 8a $(m=5000)$, different values of $p$ and $q$ will generate different demand growth patterns over the life-cycle of the new product (see Bass 1969 for details).

The optimal process change trajectories for the demand growth patterns shown in Figure $8 \mathrm{a}$ are depicted in Figure 8b. Figure 8b shows that a process with a steep demand growth and higher demand peak will require a delay (and reduction) of process change. It reflects the fact that when demand grows rapidly, the negative impact associated with low yields (caused by process change) is especially profound. Therefore, in such an environment, a firm should focus on keeping yields at a high level and delay change. After yields are stabilized at a certain level and demand growth has slowed down, potential cost savings driven by process change will outweigh the benefit of further yields improvement.

CE-observation 5 (Demand growth pattern): Production ramp-ups of processes with 
steeper demand growth and higher demand peak will benefit from a CE-ramp.

Finally, we look at the case that the firm seeks to maximize the present value of the profit stream over the life-cycle $[0, T]$ by discounting the future profit at a rate $r$. Figure $9 \mathrm{a}$ and $9 \mathrm{~b}$ illustrate the impact of a discounting rate $r$ on the optimal learning and process change policies. As we can see, higher discounting rate will lead to less learning and process change investments over time. This is simply due to the fact that with increasing $r$, the benefits of learning and process change investments over time are less valuable to the firm. However, structurally, the policies remain similar to the special case of $r=0$ analyzed above.

\section{Discussion}

Our analysis explains the inconsistency between change rates predicted by most existing models and empirical data discussed in the Introduction. In a finite planning horizon problem, most existing learning models predicted that the amount of process change should be initially at a maximum and then decrease with time. This is inconsistent with empirical observations from high-tech industries where frequently process change is delayed to a point in time when the process has reached a certain amount of maturity (McDonald 1999). This inconsistency is most visible when looking at INTEL's CE ramp strategy. Following the work of Carillo and Gaimon (2000), we incorporate a disruptive effect of process change into our analysis. This allows us to shed light on the question when a firm should avoid any process change during the ramp-up. We show that such copy-exactly ramps are beneficial if the initial understanding of the process at transfer into production is low, if the process is difficult to improve, if small modifications can have a large effect, and if the overall lifecycle is short.

One limitation of our study is its restriction to a single manufacturing plant. Studying 
ramp-up in a multi-plant system provides a promising opportunity for future research, as it brings additional reasons to follow a copy exactly ramp-up. A second opportunity for future research lies in a richer description of the product's market dynamics. Specifically, market dynamics such as new product diffusion and price erosions are of great importance during production ramp-up and would create interesting interactions with decision variables outlined in our model. Finally, following our discussion related to Figures 1 and 2, there seems to be a need to further disentangle the two concepts of process change and learning to conduct a deeper empirical study related to process change and the idea of copy-exactly ${ }^{9}$

\section{References}

Anderson, E.G.,"The Nonstationary Staff-Planning Problem with Business Cycle and Learning Effects", Management Science, 47(6), 2001, 817-832.

Argote, L. and Epple, D., "Learning Curves in Manufacturing," Science, 247(4), 1990, 920-924.

Bass, F. M. 'A new product growth model for consumer durables', Management Science, Vol. 15, No. 5, 215-227, 1969

Bhattacharya, S., V. Krishnan, and V. Mahajan, 'Managing new product definition in highly dynamic environments', Management Science, 44(11), 1998, S50-S64

Bohn, R., "Learning by Experimentation in Manufacturing", Harvard Business School, 1987, working paper 88-001.

Bohn, R. and Terwiesch, C., "The Economics of Yield-Driven Processes", Journal of Operations Management, 18(1), 1999, 41-59.

\footnotetext{
${ }^{9}$ The authors would like to acknowledge discussions with and support from the INTEL foundation. Moreover, we are grateful for comments and suggestions that we received from Christoph Loch, Rosemarie Ziednois, Lori Rosenkopf, and Nicolaj Siggelkow, as well as participants at the 2002 POMS conference.
} 
Carrillo, J. and Gaimon, C., "Improving Manufacturing Performance Through Process Change and Knowledge Creation," Management Science, 46(2), 2000, 265-288.

Chand, S., Moskowitz, H., Novak, A., Rekhi, I., and Sorger, G., "Capacity Allocation for Dynamic Process Improvement with Quality and Demand Considerations," Operations Research, 44(6), 1996, 964-975.

Dorroh, J., Gulledge, T., and Womer, N, "Investment in knowledge: A generalization of learning by experience," Management Science, 40(8), 1994, 947-958.

Eisenhardt, K. M. and Tabrizi, B. N., "Accelerating Adaptive Processes: Product Innovation in the Global Computer Industry", Administrative Science Quarterly, Vol. 40, 1995, pp 84-110.

Fine, C., "Quality Improvement and Learning in Productive Systems," Management Science, 32(10), 1986, 1301-1315.

Fine, C. and Porteus, E., "Dynamic Process Improvement", Operations Research, 37(4), $1989,580-591$.

Gaimon, Cheryl, "Planning Information Technology - Knowledge Worker Systems," Management Science, Vol. 43, No. 9, 1997, 1308-1328

Hatch, N. and Mowery, D., "Process Innovation and Learning by Doing in Semiconductor Manufacturing," Management Science, 44(11), Part 1 of 2, 1998, 1461-1477.

Hatch, N., "Future of the Learning Curve", Presentation at POMS Conference, San Francisco 2002.

Kamien, M. and Schwartz, N., Dynamic Optimization: The Calculus of Variations and Optimal Control in Economics and Management, Elsevier North-Holland, New York, 1991.

Krishnan, V, Ulrich, K. T., "Product development decisions: A review of the literature", Management Science, 47(1), 2001, 1-21.

Lapré, M.A., Mukherjee, A.S., and Van Wassenhove, L.N., "Behind the Learning 
Curve: Linking Learning Activities to Waste Reduction," Management Science (forthcoming 2001).

Levy, F.K., "Adaption in the Production Process," Management Science, 11(6), 1965, $136-154$.

Li, G. and Rajagopalan, S., "Process Improvement, Quality, and Learning Effects," Management Science, 44(11), Part 1 of 2, 1998, 1517-1532.

Mangasarian, O.L. Nonlinear Programming, Classics in Applied Mathematics, No 10, 1966

McDonald, C., "The Evolution of Intel's Copy Exactly! Technology Transfer Method", Intel Technology Journal, Q4'98.

McIvor, R., Martin, J.D., Matsuo, H., and Ng, J., "Profiting from Process Improvement in the New Semiconductor Manufacturing Environment", Technology 8 Operations Review, 1(2), 1997.

Mody, A., "Firm Strategies for Costly Engineering Learning," Management Science, 35(4), 1989, 496-512.

Pisano, G., The Development Factory, Harvard Business School Press, 1995.

Roth, A. V., Marucheck, A., Kemp, A., and Trimble, D., "The Knowledge Factory for Accelerating Learning Practices", PlanningReview, May-June 1994

Sethi, S. and Thompson, G., Optimal Control Theory: Applications to Management Science and Economics, 2nd Edition, Kluwer Academic Publishers, 2000.

Spence, A.M., "The Learning Curve and Competition," Bell Journal of Economics, Spring, 1981, 49-70.

Terwiesch, C. and Bohn, R. "Learning and Process Improvement during Production Ramp-up", International Journal of Production Economics, 70(1), 2001, 1-19.

Thomke, S., Project Dreamcast: Serious Play at Sega Enterprises Ltd, Harvard Business 
School, 1999, case study N9-600-028.

Wright, T. P., "Factors affecting the cost of airplanes", Journal of the Aeronautical Sciences, Vol. 3, 1936, 122-128

Zangwill, W.I. and Kantor, P.B., "Toward a Theory of Continuous Improvement and the Learning Curve," Management Science, 44(7), 1998, 910-920.

\section{Appendix}

Before we present the proofs, we derive the necessary conditions by the Indirect Pontryagin Maximum Principle for optimal control model with pure state constraint (see Kamien and Schwartz, 1991, Sethi and Thompson 2000). The Hamiltonian associated with the optimal control problem is,

$$
H=R(y(x(t)), k(t))-c(Q(t)) k(t)-c_{1}(q(t))-c_{2}(v(t))+\lambda(t)[\alpha v(t)-\beta q(t)]+\delta(t) q(t)
$$

where $\lambda(t)$ and $\delta(t)$ are the co-state variables associated with the trajectory equations (6) and (7), respectively. The nonnegativity constraint (9) for $Q(t)$ is guaranteed by the nonnegativity of control variable $q(t)$ and equation (7). However, the nonnegativity constraint (8) for $x(t)$ cannot be guaranteed. In order to incorporate constraint (8), we introduce the Lagrangian as following,

$$
L=H+\eta(t)[\alpha v(t)-\beta q(t)]
$$

where $\eta(t)$ is the Lagrange multiplier associated with constraint (8). The adjoint systems of the above model are shown as the following.

$$
\begin{aligned}
& \dot{\lambda}(t)=-R_{y} y^{\prime}(x(t)) \\
& \dot{\delta}(t)=c^{\prime}(Q(t)) k(t)
\end{aligned}
$$

and with the boundary conditions $\lambda(T)=0$ and $\delta(T)=0$. By the Indirect Pontryagin Maximum Principle, the optimal learning effort $v^{*}(t)$ and the optimal process change rate 
$q^{*}(t)$ need to satisfy the following necessary conditions, respectively,

$$
\begin{gathered}
\alpha[\lambda(t)+\eta(t)]=c_{2}^{\prime}\left(v^{*}(t)\right) \\
\delta(t)-\beta[\lambda(t)+\eta(t)]=c_{1}^{\prime}\left(q^{*}(t)\right)
\end{gathered}
$$

Also $\eta(t)$ needs to satisfy the complementary slackness conditions as the following,

$$
\begin{gathered}
\eta(t) x(t)=0 \\
\eta(t) \geq 0 \\
\dot{\eta}(t) \leq 0
\end{gathered}
$$

With respect to $k(t)$, the model can be solved as a static calculus problem. Therefore, the optimal production path $k^{*}(t)$ needs to satisfy the following first order condition,

$$
R_{k}\left(y(x(t)), k^{*}(t)\right)-c(Q(t))=0
$$

Equations (16), (17), (18), (19), (20)and (21) are the necessary conditions for the optimality of the model. Furthermore, by the concavity assumption of $y(x(t))$ and the convexity assumptions of $c(Q(t))$, we can see that the Hamiltonian is concave in the state variables $x(t)$ and $Q(t)$. Since the state governing equations (6) and (7) are linear in the control variables, according to Mangasarian (1966), the necessary conditions for optimality are also sufficient.

Proof of Theorem 1: To prove part (1), we will show that the optimal learning investment, $v^{*}(t)$, is nonincreasing in $x(t)$ for any given time $t$ by two steps. At first, we show the optimal learning investment is nonincreasing in $x(t)$ for $x(t)>0$. Then, we show the optimal learning investment for $x(t)=0$ is no less than the optimal learning investment for any $x(t)>0$ to complete the proof. 
The optimal learning investment, $v^{*}(t)$, needs to satisfy the necessary condition (16). If $x(t)>0$, from (18), we have $\eta(t)=0$. Therefore, (16) can be reduced to

$$
\alpha \lambda(t)=c_{2}^{\prime}\left(v^{*}(t)\right)
$$

Because $\alpha \geq 0$, by the convexity assumption of $c_{2}(v(t))$, the above equation implies $v^{*}(t)$ is increasing in $\lambda(t) . \lambda(t)$ is the marginal value of an additional unit of knowledge at time $t$. Since the objective function is concave in $x(t), \lambda(t)$ is nonincreasing in $x(t)$ that is the knowledge level at time $t$. Therefore, we can see that $v^{*}(t)$ is nonincreasing in $x(t)$.

For $x(t)=0$, from (18) and (19), we know that $\eta(t)$ is not determined, however, $\eta(t) \geq 0$. Therefore, the optimal learning investment for $x(t)=0, v_{0}^{*}(t)$, is the solution of the following necessary condition,

$$
\alpha[\lambda(t)+\eta(t)]=c_{2}^{\prime}\left(v_{0}^{*}(t)\right)
$$

Consider for $\varepsilon \rightarrow 0$ such that the difference of $\lambda(t)$ for $x(t)=0$ and $x(t)=\varepsilon$ at time $t$ is infinitely close to 0 . Let denote the optimal learning investment for $x(t)=\varepsilon$ at time $t$ as $v_{\varepsilon}^{*}(t)$, which is the solution of equation (22). Comparing the left hand sides of equation (22) and (23), we can see that $\alpha[\lambda(t)+\eta(t)] \geq \alpha \lambda(t)$. Since $c_{2}^{\prime \prime}\left(v^{*}(t)\right) \geq 0$ (Convexity), the solution of equation $(23), v_{0}^{*}(t)$, is no less than $v_{\varepsilon}^{*}(t)$ that is the solution of equation (22). Finally, by the proof for $x(t)>0$, we can conclude $v_{0}^{*}(t)$ is no less than the optimal learning investment for any $x(t)>0$. Thus, part (1) follows.

The proof of part (2) is similar to the one for part (1). We will only provide the proof for $x(t)>0$. At any given time $t$, the optimal process change rate, $q^{*}(t)$, needs to satisfy (17). If $x(t)>0$, from (18), we have $\eta(t)=0$. Then, (17) can be reduced to

$$
\delta(t)-\beta \lambda(t)=c_{1}^{\prime}\left(q^{*}(t)\right)
$$

For any given $Q(t)$ at time $t$, the lefthand side of the above equation is decreasing in 
$\lambda(t)$. Combining the convexity of $c_{1}^{\prime}(q(t))$, we can see that for any given $Q(t), q^{*}(t)$ is nondecreasing in $x(t)$. Therefore, part (2) follows.

Proof of Corollary 2: $\delta(t)$ is the marginal value of an additional unit of process change at time $t$. Since the objective function is concave in $Q(t)$, by the convexity assumption of $c(Q(t))$, we know that $\delta(t)$ is non-increasing in $Q(t)$. Thus, for $Q_{1}(t)$, in order to have the same $q^{*}(t)$ as $Q_{2}(t)$ at time $t$, it needs a smaller $\lambda(t)$ in (17). Because $\lambda(t)$ is non-increasing in $x(t)$, the corollary follows.

Proof of Theorem 2a: Differentiate both sides of (16) with respect to time $t$, we have the following equation,

$$
\alpha[\dot{\lambda}(t)+\dot{\eta}(t)]=c_{2}^{\prime \prime}\left(v^{*}(t)\right) \dot{v}^{*}(t)
$$

By the convexity assumption of $c_{2}(v(t))$, we can see that the sign of $\dot{v}^{*}(t)$ is the same as the sign of the righthand side of (25). From (14) and (20), we can see that $\dot{\lambda}(t) \leq 0$ and $\dot{\eta}(t) \leq 0$. Therefore, $\dot{v}^{*}(t) \leq 0$.

Proof of Theorem 2b: Differentiate both sides of (17) with respect to time $t$ and using (14) and (15) to eliminate $\dot{\lambda}(t)$ and $\dot{\delta}(t)$, we have the following equation,

$$
\beta\left[R_{y} y^{\prime}(x(t))-\dot{\eta}(t)\right]+c^{\prime}(Q(t)) k(t)=c_{1}^{\prime \prime}\left(q^{*}(t)\right) \dot{q}^{*}(t)
$$

Notice that $c_{1}^{\prime \prime}\left(q^{*}(t)\right) \geq 0$. Therefore, the sign of $\dot{q}^{*}(t)$ is the same as the sign of the lefthand side of $(26)$. Because both the revenue function and $y(x(t))$ are increasing functions, combining with (20), the first term of the lefthand side of (26) is nonnegative. Since $c(Q(t))$ is a decreasing function and $k(t) \geq 0$, the second term of the lefthand side of (26) is nonpositive. Therefore, $\dot{q}^{*}(t) \geq(\leq) 0$ if $\beta\left[R_{y} y^{\prime}(x(t))-\dot{\eta}(t)\right]+c^{\prime}(Q(t)) k(t) \geq(\leq) 0$.

Proof of Theorem 2c:The optimal output rate $k^{*}(t)$ should satisfy (21). Differentiate both sides of (21) with respect to time and using (7) to eliminate $\dot{Q}(t)$, we have,

$$
-R_{k k} \dot{k}(t)=R_{k y} y^{\prime}(x(t)) \dot{x}(t)-c^{\prime}(Q(t)) q(t)
$$


By the concavity and supermodular assumptions of the revenue function, we know that $R_{k k} \leq 0$ and $R_{k y} \geq 0$. Therefore, the sign of $\dot{k}(t)$ is the same as the sign of the righthand side of $(27)$. If $\dot{x}(t) \geq 0$, the righthand side is always nonnegative. That implies $k(t)$ increases over time. However, if $\dot{x}(t) \leq 0, k(t)$ increases (decreases) over time if $R_{k y} y^{\prime}(x(t)) \dot{x}(t)-c^{\prime}(Q(t)) q(t) \geq(\leq) 0$.

Lemma 1 (Properties of the co-state variables)

The co-state variables, $\lambda(t)$ and $\delta(t)$, are nonnegative non-increasing over time.

Proof of Lemma 1: Because $R(y(x(t), k(t))$ is increasing in $y(x(t)), y(x(t))$ is increasing in $x(t)$, and $c(Q(t))$ is decreasing in $Q(t)$ by assumptions, the above lemma follows from differential equations (14) and (15) and the boundary conditions $\lambda(T)=0$ and $\delta(T)=0$.

Proof of Theorem 3: Solving the first-order differential equations (14) and (15), we have the following expressions for $\lambda(t)$ and $\delta(t)$,

$$
\begin{gathered}
\lambda(t)=\int_{t}^{T} R_{y} y^{\prime}(x(s)) d s \\
\delta(t)=-\int_{t}^{T} c^{\prime}(Q(s)) k(s) d s
\end{gathered}
$$

Substitute (28) into (16) and differentiate both side of (16) with respect to $\alpha$, we have,

$$
\lambda(t)+\eta(t)=c_{2}^{\prime \prime}\left(v^{*}(t) \frac{d v^{*}(t)}{d \alpha}\right.
$$

where $\lambda(t)$ and $\delta(t)$ are expressed in (28) and (29), respectively. Therefore, by lemma 1 , (19), and convexity of $c_{2}(v(t))$, we can see $\frac{d v^{*}(t)}{d \alpha} \geq 0$.

From (6), we have $\alpha=\frac{\dot{x}(t)+\beta q(t)}{v(t)}$. Substitute it, (28), and (29) into (16) and differentiate both side of (16) with respect to $\beta$, we have,

$$
[\lambda(t)+\eta(t)] \frac{q(t)}{v^{*}(t)}=\frac{\partial v^{*}(t)}{\partial \beta}\left[c_{2}^{\prime \prime}\left(v^{*}(t)\right)+\frac{\dot{x}(t)+\beta q(t)}{v^{*}(t)^{2}}(\lambda(t)+\eta(t))\right]
$$

Thus, by lemma 1, (19), convexity of $c_{2}(v(t))$, and the nonnegativity of $q(t)$ and $v(t)$, we have that $\frac{\partial v^{*}(t)}{\partial \beta} \geq 0$. 
Substitute (28) and (29) into (17) and differentiate both side of (17) with respect to $\beta$, we have

$$
-[\lambda(t)+\eta(t)]=c_{1}^{\prime \prime}\left(q^{*}(t)\right) \frac{d q^{*}(t)}{d \beta}
$$

Therefore, by lemma $1,(19)$, and convexity of $c_{1}(q(t))$, we have $\frac{d q^{*}(t)}{d \beta} \leq 0$.

From (6), we have $\beta=\frac{\alpha v(t)-\dot{x}(t)}{q(t)}$. Substitute it, (28), and (29) into (17) and differentiate both side of (17) with respect to $\alpha$, we have

$$
[\lambda(t)+\eta(t)] \frac{v(t)}{q^{*}(t)}=\frac{\partial q^{*}(t)}{\partial \alpha}\left[\frac{\alpha v(t)-\dot{x}(t)}{q^{*}(t)^{2}}(\lambda(t)+\eta(t))-c_{1}^{\prime \prime}\left(q^{*}(t)\right)\right]
$$

Although $\lambda(t)+\eta(t), v(t)$, and $q^{*}(t)$ are all positive, the sign of $\frac{\alpha v(t)-\dot{x}(t)}{q^{*}(t)^{2}} \lambda(t)-c_{1}^{\prime \prime}\left(q^{*}(t)\right)$ is not determined. Therefore, the sign of $\frac{\partial q^{*}(t)}{\partial \alpha}$ could be positive or negative.

Proof of Theorem 4: The Hamiltonian associated with the above optimal control model is,

$$
H=-c_{1}(q(t))-c_{2}(v(t))+\lambda(t)[\alpha v(t)-\beta q(t)]+\delta(t) q(t)
$$

where $\lambda(t)$ and $\delta(t)$ are the co-state variables associated with the trajectory equations. We introduce the Lagrangian to incorporate the state constraint (8).

$$
L=H+\eta(t)[\alpha v(t)-\beta q(t)]
$$

The corresponding adjoint systems of the above model have the following format,

$$
\begin{aligned}
& \dot{\lambda}(t)=-\frac{\partial L}{\partial x(t)}=0 \\
& \dot{\delta}(t)=-\frac{\partial L}{\partial Q(t)}=0
\end{aligned}
$$

with the boundary conditions $\lambda(T)=\theta$ and $\delta(T)=\xi$.

By the Indirect Pontryagin Maximum Principle for pure state constraints, the optimal learning effort $v^{*}(t)$ and the optimal process change rate $q^{*}(t)$ need to satisfy the following necessary conditions that have the same forms as (16) and (17), respectively,

$$
\alpha[\lambda(t)+\eta(t)]=c_{2}^{\prime}\left(v^{*}(t)\right)
$$




$$
\delta(t)-\beta[\lambda(t)+\eta(t)]=c_{1}^{\prime}\left(q^{*}(t)\right)
$$

The adjoint systems (33), (34), and the boundary conditions imply that $\lambda(t)=\theta$ and $\delta(t)=\xi$, i.e., both co-state variables are constants over the whole time horizon $[0, T]$. If $x(t) \geq 0$, for all $t \in[0, T]$, we have $\eta(t)=0$. Therefore, from (35) and (36), Theorem 4 follows. 


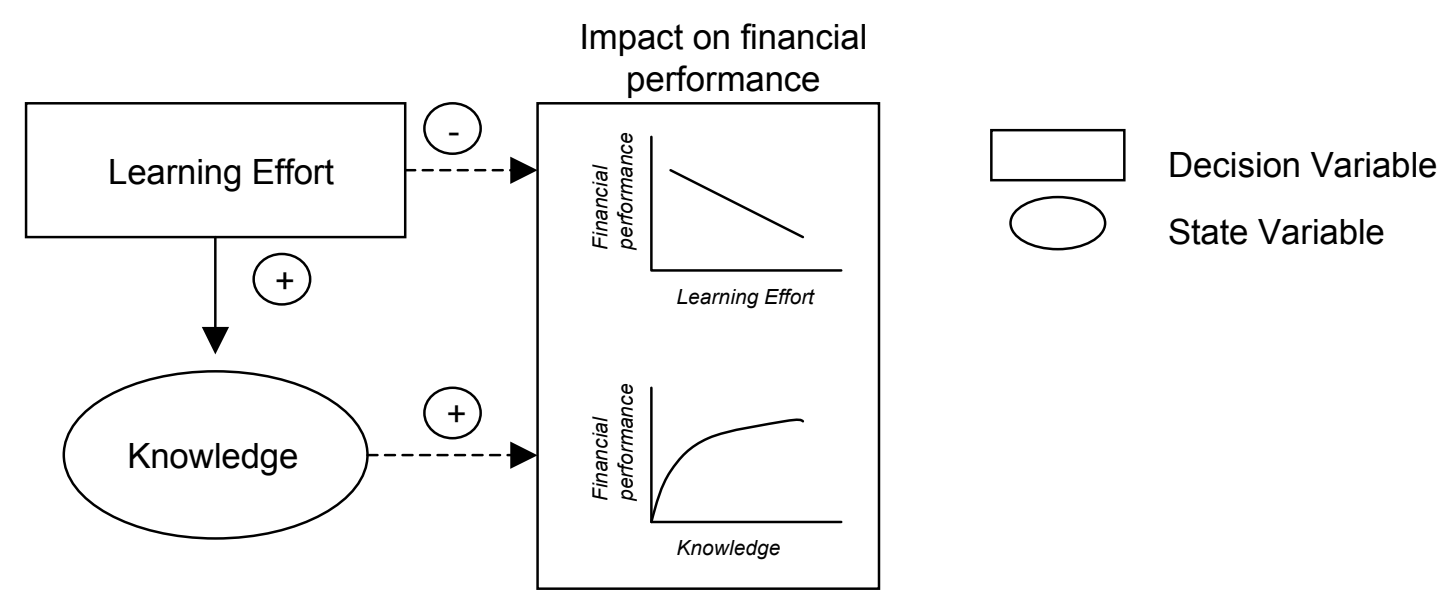

Figure 1: The elements of learning curve

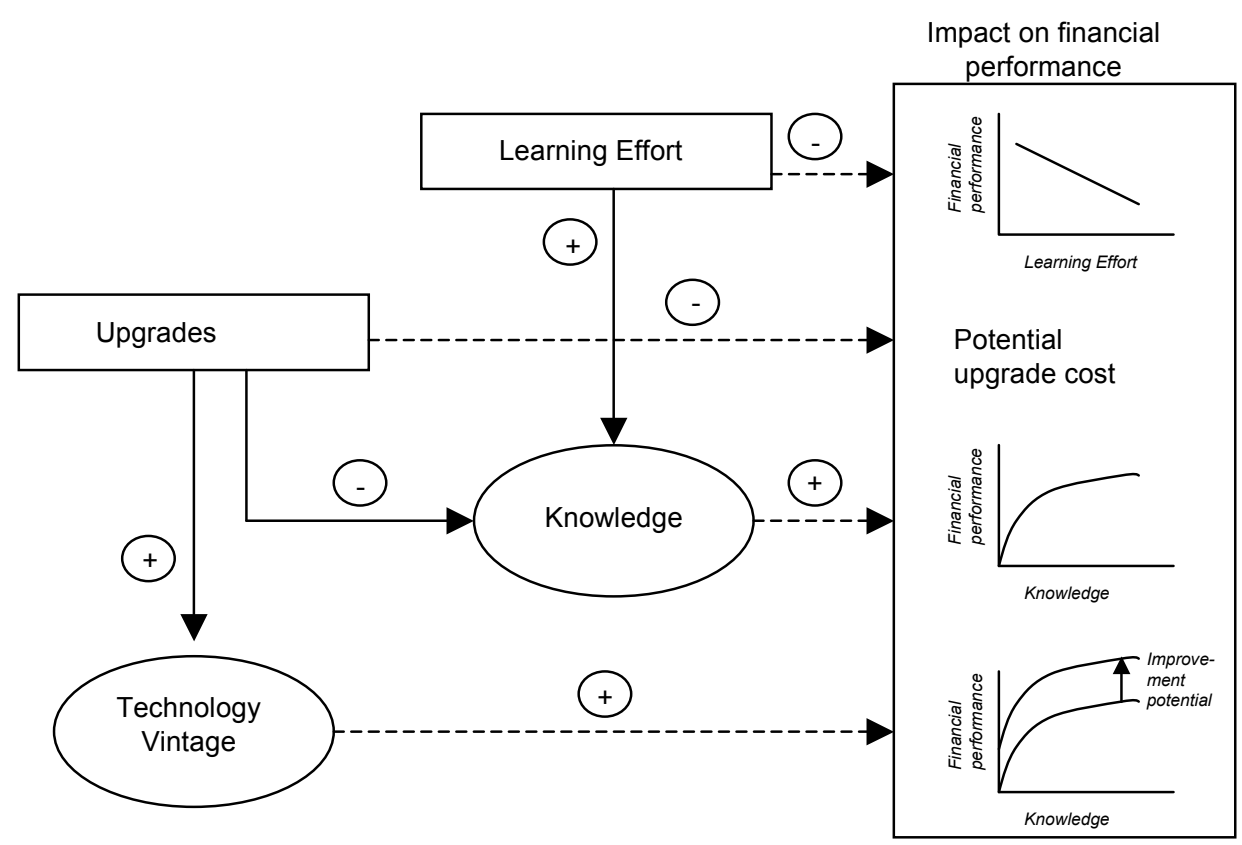

Figure 2: Theoretical framework of the model 


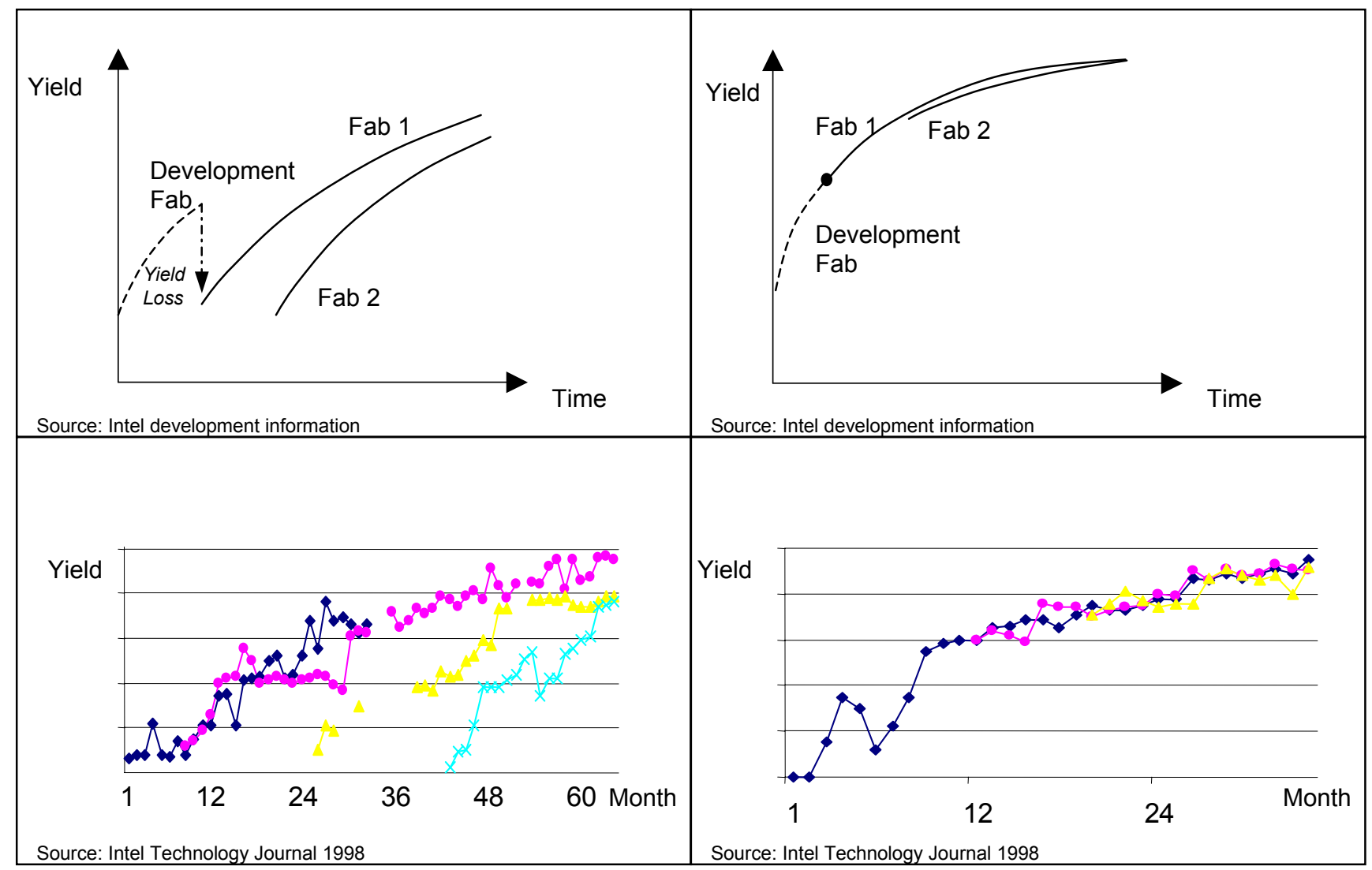

Figure 3: The basic idea behind INTEL's copy-exactly ramp-strategy: Theory (upper part) and empirical evidence (lower part)
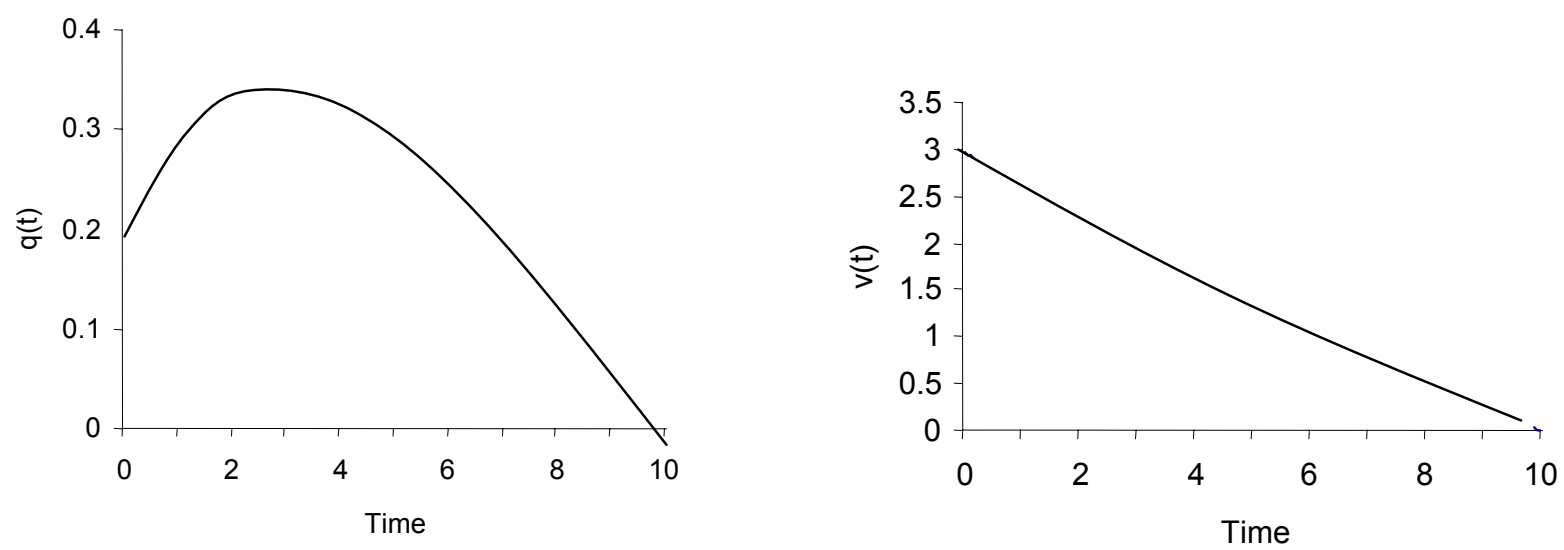

Figure 4ab: Optimal process change and learning trajectory for the base case 

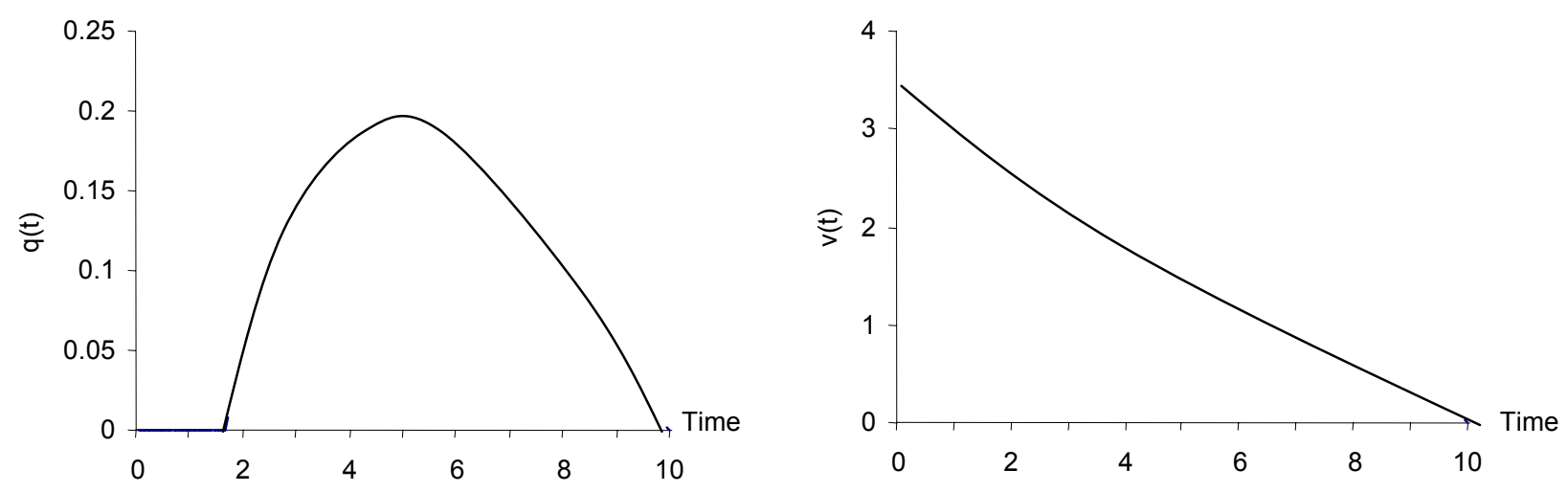

Figure 5ab: Optimal process change and learning trajectory for $x(0)=2$
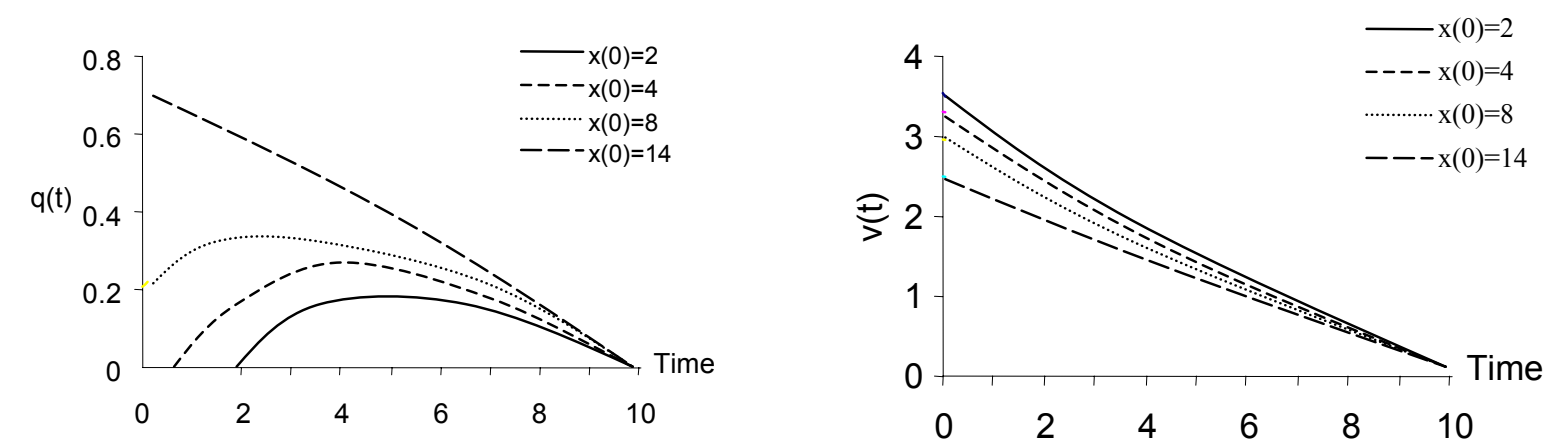

Figure 5cd: Impact of $x(0)$ on the optimal process change and learning trajectories
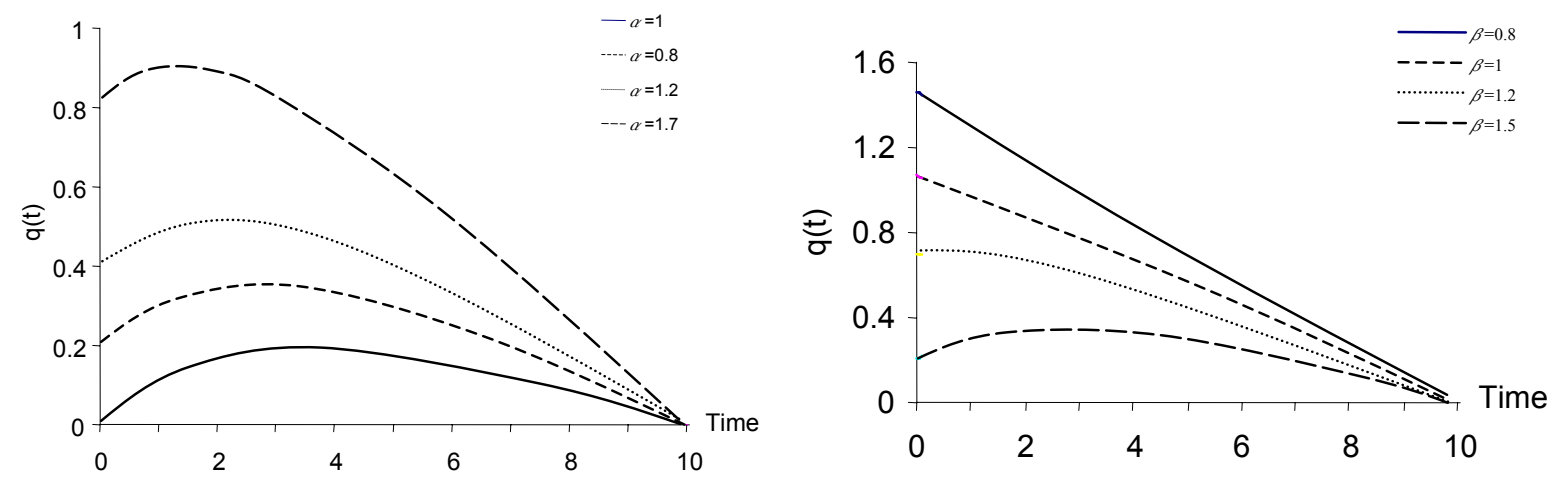

Figure 6ab: Impact of learning parameter $\alpha$, disruption parameter $\beta$ on the optimal process change trajectories
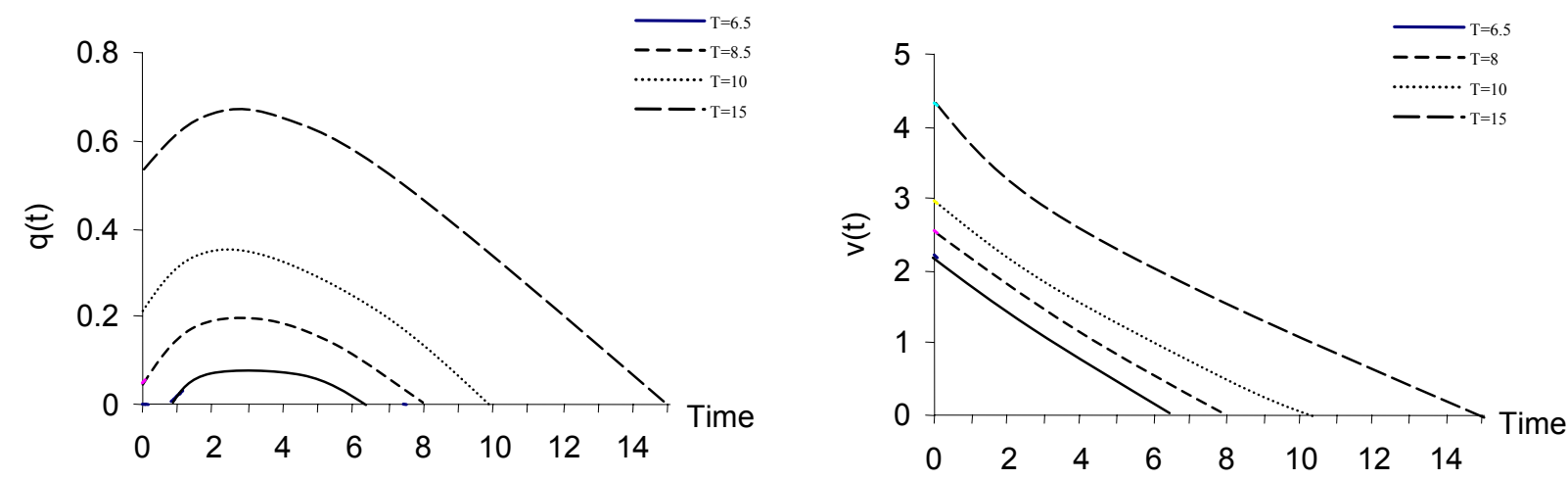

Figure 7ab: Impact of lifecycle length $T$ on the optimal process change and learning trajectories 

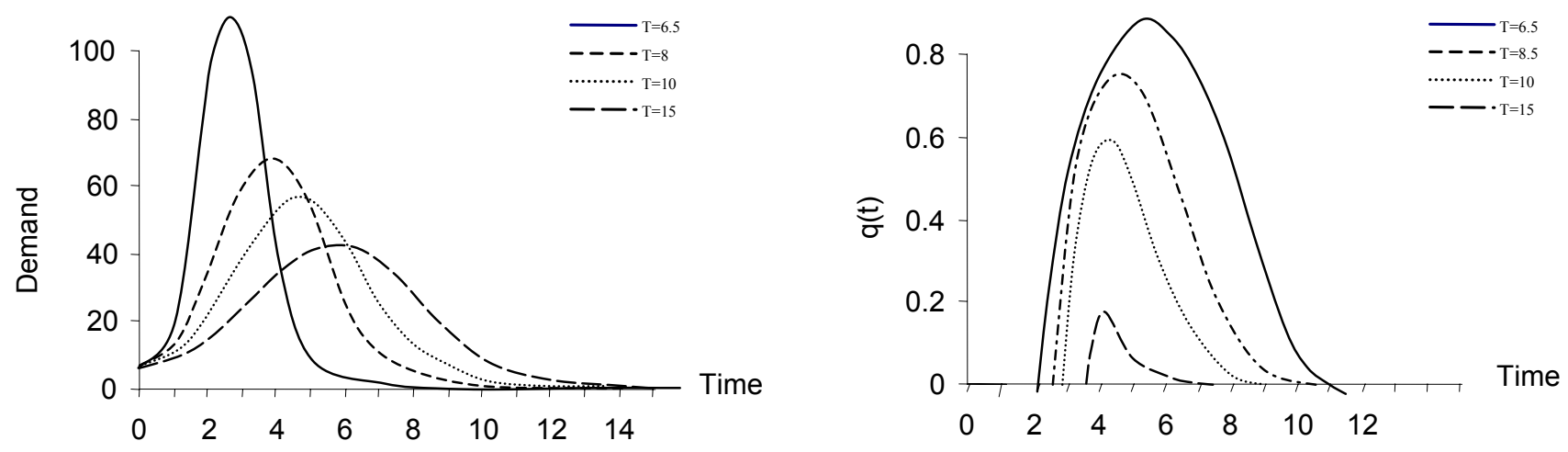

Figure 8a:Bass Diffusion

Figure 8b:Impact of product lifecycle on the optimal process change trajectory
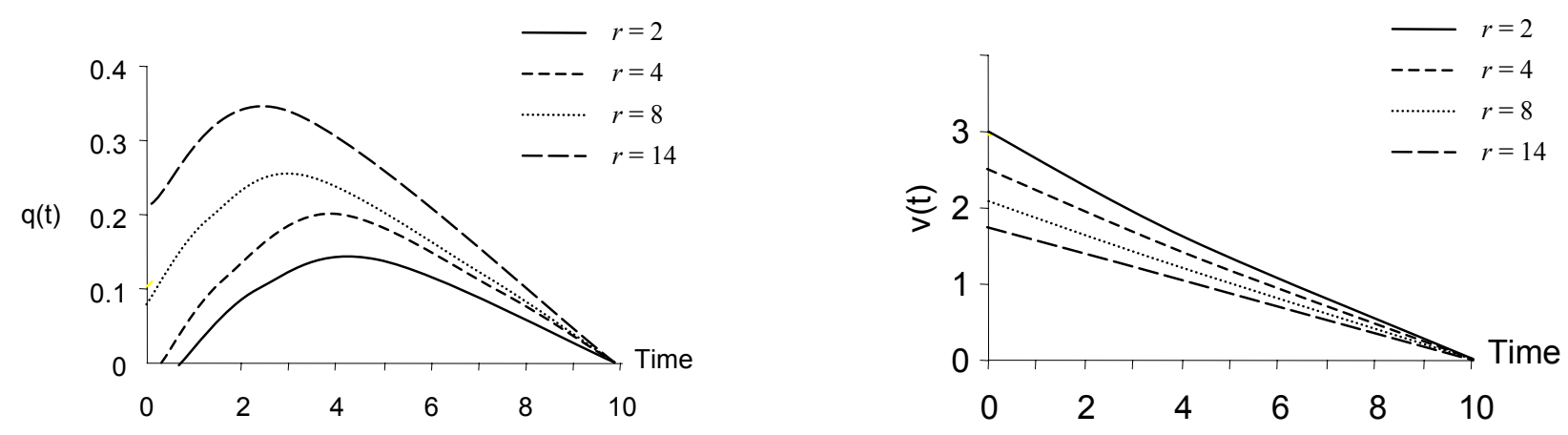

Figure 9ab: Impact of discount rate on the optimal process change and learning trajectories 
Table 1: Summary of Notation

\begin{tabular}{ll}
\hline \multicolumn{1}{c}{ Notation } & \multicolumn{1}{c}{ Description } \\
\hline$T$ & Time; $t \in[0, T]$, the product life-cycle length is $T$ \\
$v(t)$ & Rate of learning at time $t ; v(t) \in[\underline{v}, \bar{v}]$. \\
$q(t)$ & Rate of process change at time $t ; q(t) \in[\underline{q}, \bar{q}]$. \\
$k(t)$ & Rate of production at time $t ; k(t) \in[0, \infty]$. \\
$x(t)$ & Knowledge level at time $t$. \\
$Q(t)$ & Cumulative process change at time $t$. \\
$x_{0}$ & Initial knowledge level at time 0. \\
$\alpha$ & Impact on knowledge level per unit of learning investment. \\
$\beta$ & Impact on knowledge level per unit of process change investment. \\
$y(x(t))$ & Yield level as a function of the knowledge level at time $t$. \\
$p(d(t))$ & Price of the product at output level $d(t)$. \\
$R(\mathrm{y}(x(t)), k(t))$ & Revenue as a function of the yield level and production rate at time $t$. \\
$c(Q(t))$ & Unit manufacturing cost as a function of the cumulative process change at time $t$. \\
$c_{1}(q(t))$ & Cost of $q$ unit of process change at time $t$. \\
$c_{2}(v(t))$ & Cost of $v$ unit of learning at time $t$. \\
\hline
\end{tabular}

Table 2: Summary of Assumptions

\section{Assumptions:}

Structure Assumptions:

A1: Yield, $y(x(t))$, is an increasing and concave function of the knowledge level at time $t$.

A2: Unit manufacturing cost, $c(Q(t))$, is a decreasing and concave function of the cumulative process change at time $t$.

A3: The firm faces a stationary downward sloping demand curve, $p(d(t))$.

Analytical Assumptions:

A4: Cost of process change at time $t, c_{1}(q(t))$, is a increasing and convex function of $q(t)$.

A5: Cost of learning at time $t, c_{2}(v(t))$, is a increasing and convex function of $v(t)$.

A6: The revenue at time $\mathrm{t}, R(y(x(t)), k(t))$, is a concave and super-modular function in $y(x(t))$ and $k(t)$. 